\title{
Captures entomologiques
}

Jean Leclercq, Marcel Leclercq

\section{Citer ce document / Cite this document :}

Leclercq Jean, Leclercq Marcel. Captures entomologiques. In: Bulletin de la Société entomologique de France, volume 48 (1),1943. pp. 12-13;

https://www.persee.fr/doc/bsef_0037-928x_1943_num_48_1_15638

Fichier pdf généré le 25/06/2019 


\title{
Captures entomologiques
}

\author{
par J. el M. Lecierce
}

Nous signalerons ici quelques captures effectuées naguère, pendant de? brefs séjours dans le nord de la France : départements de: l'Eure, du Nord et dans le IIainaul $\left({ }^{1}\right)$.

A Fains, près de Pacy (Eure), du 16.V. an 3.VI.40, nous avons noté, comme formant la faune entomologique des sentiers:

Coléoptìnes. - Timarcha lenebricosa F., Clytra quadripanclala I., Pyrrochroa purpurala Mull. et Trichodes alvearius F.

Hyménoptèris. - Alomya debellalor F. ot Nomada fulvicornis F., 우.

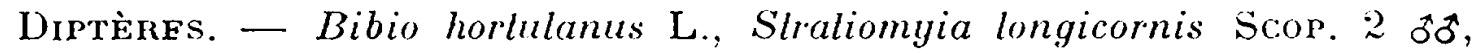
Diortria rufipes Deg., Argyramoeba anlhrax Scmk., Chilosia variabilis Panz. ठૈ, Merodon clavipes F. abondant, les ỡ sur les fleurs d'Ombelliféracées, les qo posées par terre, dans les chemins ; Criorrhina floccosa MG. \&, Chrysoloxum festivum L. \&, Dorycera graminum F., Sarcophaga agnala Rond. o, enfin Fabricia ferox PANz.

A Vernon (Eure), 21.V.40:

Diptères. - Xylota lenla MG. ô el de nombreux Ortalis gullata MG.

A Thun-Saint-Martin, près de Cambrai (Nord), 25, 26 et 27.V.40, le long des haies et dans les prairies humides:

Conéoptènes. - Cantharis fusca L., Anlhocomus equestris F., Cyphon coarclatus PaYk., Oedemera lurida Maksh., Pyrrochroa cacharias Muls., Chrysomela polita L., Celonia aurala L. et Silpha alrala L. Tous ces Coléoptères ont été pris sur les ombelles de Chaerefolium silvestre Scr. Tr.

Hyménoptères. - Quelques Ichneumonides : Clislop!yga incilalor F. 우,

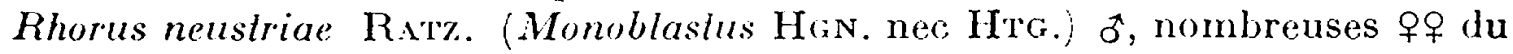
Diplazon laelatorius F. et d'Alnilasta? caedalor Gr.

Les Vespiformes et Apides suivants abondaient sur les piquels de clôture, dans les prés: Trypoxylon figulus I., Sapyga clavicornis L. et (moins souvent.) S. quinquepunclala F., Chrysis cyanea L. et ignila L., Eriades maxillosus L., Osmia parvala D. el P. ot Osmia venlralis Panz.

DIPTÈRES. - Telanocera ferruginéa FA.L.

A Leuze (Hainaut), 15.V.10:

Hyménoptènes. - Caloglyplus forlipes Gr. o, P'aniscus ocellaris Thoms. ô, Ichneumonide intéressant, dérit de la suede et probablement nouveau pour la faune française, rar il n est pas mentionne au Catalogue .J. IJ Gavlle (1908), Odynerus (Ancistrocertes) oriventris Wes.r. o, Nomada fabriciana L. $q$ et Eucera longicornis L. + , ce dernier pris sur une fleur de Ranunculus it ait anormalement pelit.

(1) Nous avons dû recourir pour certaines identifications, ì la competence de MM. J. I).

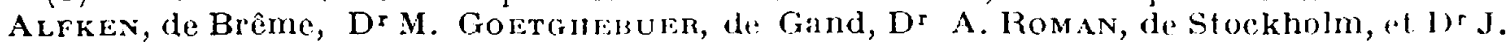
Villentuve de Janti, de Rambouillet. 
A Pommereoul (Hainaut), 2:2.IX.17:

Un o du Microcryptus nigrocinclus Gr. est sorti d'une chrysalide de Pyrameis alalanla L. élevée par A. Dufrane. Cette Cryptine manque au Catalogue J. de G.uulle et doit être nouvelle pour la faune française.

Enfin, rapportons la capture d'une + , du Parabalus lalungula Tiss. à Virton (Luxembourg), 26.V.38., celle d'une ò d'Amblyleles quadripunclorius Muli. (bidenlorius F.) à Arlon, VIII.37 et celle d'un ot de Lissonola cf. carbonaria Holmg. a Carslbourg (Lux.), 20.VIII.1882. Cetle dernière forme est, semblet-il, fort rare, sauf en Scandinavie.

\title{
Un problème de nomenclature au sujet des Noterus clavicornis et crassicornis
}

[COL. Dytiscidate]

\author{
par le Dr F. GurgnoT
}

Le genre Nolerus comprend en Europe trois espèces depuis longtemps connues, mais si tout le monde est d'accord sur l'une d'elles : laevis Sturm, il est loin d'er être de même pour les deux autres. Leur synonymie apparaît très embrouillée pour un double motif : d'abord, parce qu'ayant été successivement décrites plusieurs fois el d'une manière très sommaire, on ne savait plus trop à laquell. des deux espèces chaque nom devait être attribué, ensuite parce que certains des anciens auteurs ont indiqué presque dès le début des synonymies incorrectes. L'accord paraissait établi à la suile de la publication du Coleoplerorum Calalogus, Dyliscidae, par Zimmenmann qui avait adopté le nom de clavicornis de Geer pour la grande espèce et celui de crassicornis Müller pour la plus petite, opinion que j'avais moi-même suivie (1). Mais dans une étude récente M. Fr. Balfolin-Browne (2) vient de remettre tout en question en proposant une interprétation diamétralement opposée, qui avait été déjà celle de Des Gozıs ( ${ }^{\mathbf{3}}$ ). Voyons s'il y a possibilité de résoudre cette difficulté.

Pour cela un moyen se presente tout d'abord naturellement à l'esprit : examiner les types des premiers descripteurs, De GeEr el Müller, pour voir à quelles espèces s'appliquent les noms qu'ils ont donnés. Malheureusement ces lypes sont introuvables et cetle constatation devient impossible.

ll ne reste done que la ressource de rechercher la solution par le raisonnement et la confrontation des premiers textes. Nous possédons heureusement deux points de repère pour identifier ces espèces : l'une est plus grande $(4,2$ à $4,7 \mathrm{~mm}$.) et a chez les ot les antennes assez progressivement dilatées, un peu

(1) F. Gugnot, Hydrocanthares de firance, 1931-33, p. 49.4 et suis.

(2) Fr. Balfoth-Browne, Enl. Montill. Mag., 70, 1934, p. 12s.

(3) Des Go7.1s, Miscell. Ent., 21, 1913, p. s2 et 83. 